\title{
Relationship between Social Support and Life Satisfaction of College Students: Resilience As a
}

\section{Mediator and Moderator}

\author{
Yuan Guo
}

(Guangdong University of Foreign Studies, 641663882@qq.com)

\section{Introduction}

In recent years, with the rise of positive psychology, research on subjective well-being and life satisfaction has attracted the attention of scholars. Life satisfaction is increasingly an important dimension of subjective well-being. It refers to the subjective evaluation of quality of life based on the standard set by individuals. It is an important parameter to measure in people's lives (Ou 2017). As the main indicator of individual subjective well-being, life satisfaction is also a general evaluation of the quality of life of a person according to their own choice criteria (Ye \& Lu 2017). Previous studies have shown that life satisfaction is closely related to social support. There is a direct correlation between levels of social support and subjective perceived individual life satisfaction (Feng \& Wan 2016; Cui \& Yao 2012; Ma \& Wang 2013; Yu et al. 2013).

Social support refers to the influence of a person through social contact to alleviate psychological stress reaction, relieve mental tension, and improve social adaptability (Li 1998). It can stimulate the positive emotional experience of individuals, give them psychological comfort and courage, and help people more actively cope with difficulties. It is beneficial to the stability of mood, peace of mind, and physical and mental health of individuals. Many studies have found that good social support is positively correlated with life satisfaction and positive emotions but is negatively correlated with loneliness, depression, anxiety and other negative emotions (Yang 2009). For example, Ratelle, Simard and Guay 
(2013) found that support from parents, friends, and lovers was positively correlated with the subjective well-being of college students. A survey of more than 800 adolescents by Wang, Li and Mu (2016) found that social support had a significant predictive effect on life satisfaction. Social support buffer model theory holds that social support not only directly affects the physical and mental health of the individual but also maintains the physical and mental health of the individual through the adjustment of other factors. Baron and Kenny (1986) argued that there is a mediating variable between social support and well-being that plays a driving role between the independent variable (social support) and dependent variable (well-being). However, others hold that the relationship between external environment, internal factors, and life satisfaction is very complex; that this relationship will become the focus of future life satisfaction research (Chen \& Zhang 2013). The study of Adela (2001) provides us with an important variable: self-esteem. Zhang and Xing (2007) in China also believe that social support not only directly affects life satisfaction, but also impacts subjective well-being through interactions with internal factors such as individual self-esteem, psychological capital, self-control, depression, and loneliness. Li (2010) concluded that self-consistency and congruence played a fully intermediary role between social support and life satisfaction after investigating 360 college students. Wang, $\mathrm{Li}$ and $\mathrm{Mu}$ (2016) found that adolescents' positive emotions held a mediating role in the relationship between social support and life satisfaction. From the perspective of positive psychology, Wang's (2014) survey of 5 universities found that positive psychological capital can directly affect life satisfaction. Wang also found that positive psychological capital can indirectly affect life satisfaction through the mediating role of social support. Yang (2009) states that core self-evaluation is an intermediate variable between social support and subjective well-being. Recently, studies have explored the mediating effects of self-esteem, positive emotions, psychological capital, depression and self-control, self-consistency and congruence, and core self-evaluation. As an important psychological factor, resilience may also play an intermediary role between social support and life satisfaction.

In recent years, resilience has become an active topic in the field of positive psychology. Resilience refers to a relatively stable psychological 
trait of individuals to maintain and promote healthy growth when coping with adversity and stress (Feng 2016). In 2005, the American Psychological Association (APA) defined resilience as adapting well in the face of adversity, trauma, grief, threats, or other major stresses (Ou 2017). Studies have shown that resilience can predict well-being. High resilience individuals can maintain happiness in the face of adversity, continue to pursue and achieve goals, and make life happy and meaningful (Wang \& Wang 2013). Fredrickson et al. (2008) found that increasing resilience predicts the increase of life satisfaction and the decrease of depressive symptoms. Feng (2016) also found that both social support and resilience have a positive predictive effect on personal life satisfaction. Pietrzak et al. (2010) found that resilience had a moderating effect on social support and PTSD and depressive symptoms. They also found that individuals with high resilience can better utilize their own social support system to overcome difficulties and adapt to changing environments so as to protect their mental health. Sun et al. (2013) found that social support and resilience have protective effects on the emotional and behavioral problems of youths; and that resilience plays a mediating and moderating role on the effect of social. Resilience may also enhance the protective effects of social support on life satisfaction. Therefore, this study proposes that resilience plays a moderating role between social support and life satisfaction.

This study surveys college students. The findings provide empirical evidence supporting the relationship between social support, resilience and life satisfaction.

\section{Method}

The research method in this study mainly included three steps, sampling of subjects, use of scales and data analysis, which has been widely applied to research fields such as sociology, moral education and psychology (Skoe 2010; Malti et al. 2013).

\subsection{Participants}

The subjects completed a consent form prior to the study which was approved by Guangdong University of Foreign Studies. 900 
questionnaires were sent out to college students in Guangdong University of Foreign Studies. 886 questionnaires were recovered. Of these, 843 were valid questionnaires. The effective rate of the samples was about 95\%. Respondents consisted of 464 males and 379 females. 298 respondents were from single-child households, 545 were not; 489 were urban students, 354 were rural students; 413 were liberal arts students, 209 were science students, and 221 were engineering students. The average age of respondents was 21.6 years old ( $\mathrm{SD}=0.92)$.

\subsection{Measures}

\section{Resilience scale}

The resilience scale, compiled by $\mathrm{Hu}$ and Gan (2008), comprises a total of 27 questions and uses a 5 points scale (1=not meet at all, $5=$ full compliance); the higher the total score, the higher the level of psychological resilience. The scale is divided into two dimensions: individual force and support force. The individual force considers three dimensions: goal concentration, emotion control, and positive cognition. The support force considers two dimensions: family support and interpersonal assistance. In this study, the internal consistency reliability of individual force and support force is 0.80 and 0.67 , respectively. The internal consistency reliability of each dimension is as follows: goal concentration 0.685 , emotion control 0.754 , positive cognition 0.736 , family support 0.757 , and interpersonal assistance 0.714 . The overall internal consistency reliability is 0.87 .

\section{Social support scale}

The social support scale, compiled by Xiao (1994), comprises a total of 10 questions. These consider three dimensions: objective support, subjective support, and the use of social support. Objective support refers to visible practical support including material support, social networks, the existence and participation of group relationships. A higher objective support score indicates that an individual has more real direct support. Subjective support refers to the emotional experience that an individual has when they feel respected, supported, and understood in society. The higher the subjective support scores, the more the individual feels 
respected, supported and understood in society. The degree of social support utilization refers to individual differences in the use of social support. This has a certain objective basis; the higher the score, the higher the individual's degree of social support. Since all the respondents were college students, some scale items require revision. For example, "colleague" is changed to "classmate," "neighbor" is changed to "roommate," and "spouse" is changed to "companion." The revised social support scale still maintains the three-dimensional structure of the original scale and was retested at a 2-week interval. The test-retest reliability is 0.92 . The internal consistency coefficient of each dimension of the scale is $0.85-0.91$. In this study, the total $\alpha$ coefficient is 0.86 .

\section{Life satisfaction scale}

The life satisfaction scale, compiled by Pavot et al. (1991) to investigate college students' satisfaction with their current life, comprises a total of 5 self-assessment items. A 1-7 grade scoring method is adopted. A 1 indicates "strongly disagree" while a 7 indicates "strongly agree;" the higher the score, the higher the life satisfaction. The internal consistency reliability of the scale for our data was 0.84 , and showed good reliability and validity index.

The SPSS ${ }^{\circledR} 21.0$ and Amos 17.0 were used for data analysis in this study.

\section{Results}

\subsection{Correlation analysis of social support, resilience and life satisfaction}

Table 1 lists the Pearson correlation coefficients among resilience, life satisfaction, total scores of social support, and each of its dimensions. There is a significant positive correlation between resilience and social support (and its dimensions), and between resilience and life satisfaction. Subjective support, objective support and support utilization are significantly and positively correlated with resilience and life satisfaction ( $\mathrm{r}=0.350, \mathrm{p}<0.001 ; \mathrm{r}=0.353, \mathrm{p}<0.001 ; \mathrm{r}=0.328, \mathrm{p}<0.001 ; \mathrm{r}=0.345, \mathrm{p}<0.001$; $\mathrm{r}=0.370, \mathrm{p}<0.001 ; \mathrm{r}=0.350, \mathrm{p}<0.001)$. The total scores of social support are also significantly and positively correlated with resilience and life satisfaction $(\mathrm{r}=0.412, \mathrm{p}<0.001 ; \mathrm{r}=0.417, \mathrm{p}<0.001)$. The results show that 
greater social support and resilience correlate with an increase in life satisfaction. However, if college students do not have good psychological resilience, they cannot adapt well when facing difficulties and pressure. In such cases, even if strong social support is present, life satisfaction remains relatively low. Resilience is significantly and positively correlated with life satisfaction $(\mathrm{r}=0.518, \mathrm{p}<0.001)$. The results show that greater resilience correlates with an increase in life satisfaction.

\begin{tabular}{llllll}
\hline 1 os & $2 \mathrm{ss}$ & $3 \mathrm{su}$ & $4 \mathrm{ts}$ & $5 \mathrm{r}$ & $6 \mathrm{ls}$ \\
\hline
\end{tabular}

11

$20.571^{* * *} \quad 1$

$3 \quad 0.544^{* * *} \quad 0.582^{* * *} \quad 1$

$4 \quad 0.868^{* * *} \quad 0.825^{* * *} \quad 0.830^{* * *} \quad 1$

$5 \quad 0.327^{* * *} \quad 0.350^{* * *} \quad 0.374^{* * *} \quad 0.413^{* * *} 1$

$6 \quad 0.344^{* * *} \quad 0.364^{* * *} \quad 0.349^{* * *} \quad 0.416^{* * *} \quad 0.517^{* * *} 1$

Table 1. Correlation analysis among social support, resilience and life satisfaction variables $(n=843)$

Note : ${ }^{*} P<0.05,{ }^{* *} P<0.01,{ }^{* * *} P<0.001$

os=objective support ss=subjective support su=support utilization

ts= total scores of social support $\quad r=$ resilience $\quad l s=$ life satisfaction

According to Tsui et al. (1995), the correlation level critical value is generally above 0.75 . In this study, with the exception of a high social support and its subscales correlate, correlation coefficients were no more than 0.6. There was no serious multicollinearity problem among the major variables.

3.2 Analysis of the mediating effect of resilience on social support and life satisfaction

A Structural Equation Model (Wu 2010), shown in Figure 1, is constructed using social support as the independent variable, psychological resilience as the mediating variable, and life satisfaction as the dependent variable. According to Wu \& Wen's (2011) project package 
recommendations, the life satisfaction dimension scale is packaged using a factor method. Two indicators (life satisfaction A, life satisfaction B) are obtained. Using Amos17.0 (Wu 2010) to fit the mediation model, the fitting indexes of the model are: $\mathrm{X}^{2} / \mathrm{df}=6.719, \mathrm{GFI}=0.980, \mathrm{NFI}=0.974$, $\mathrm{CFI}=0.978, \mathrm{IFI}=0.978, \mathrm{RMSEA}=0.070$. Each index is above 0.9 and RESEA is less than 0.08 . The fitting degree of the model is comparatively ideal.

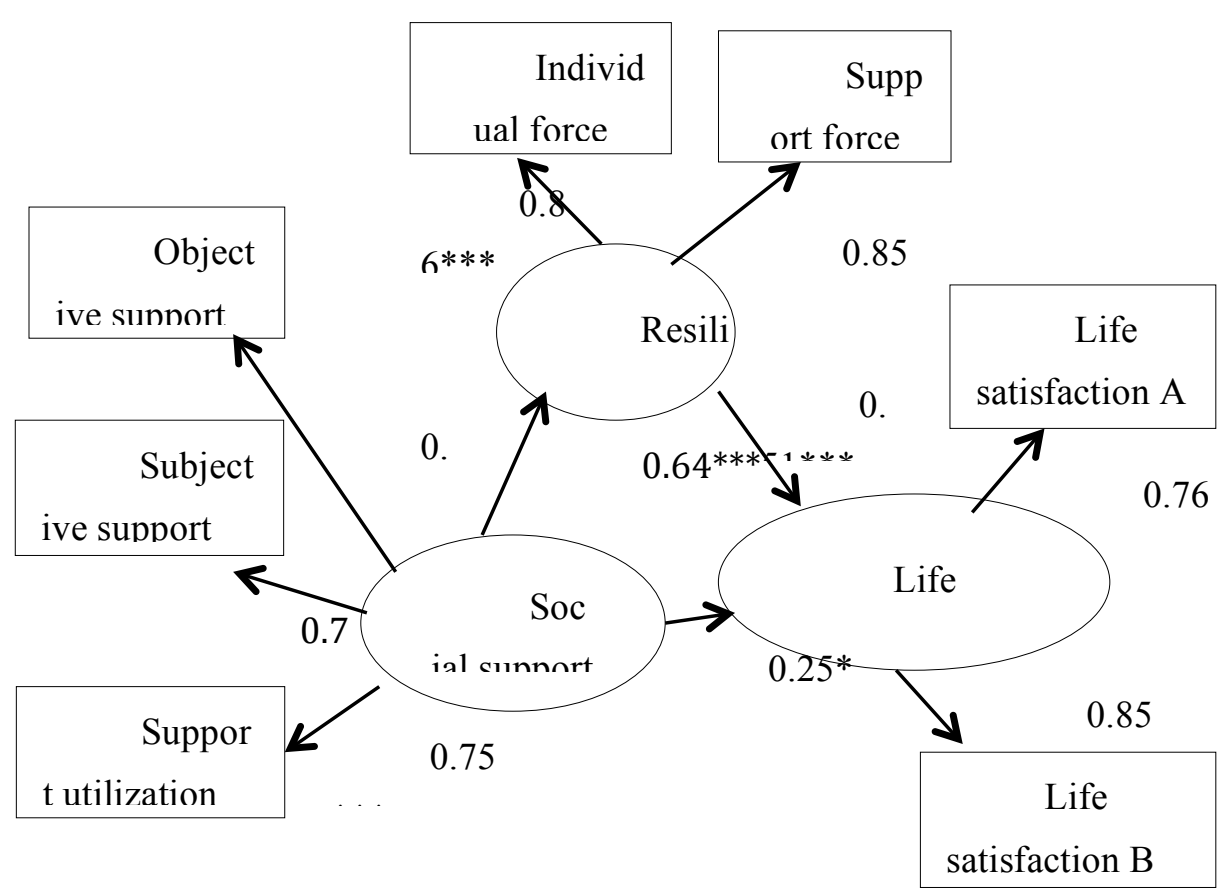

Figure1. The path diagram of the mediating effect of resilience on social support and life satisfaction

The results show that the path coefficients of social support, resilience and life satisfaction are significant. Social support not only directly affects life satisfaction, but also indirectly affects life satisfaction through the mediating effect of resilience. The direct effect and indirect effect is $0.254(\mathrm{P}<0.001)$ and 0.325 , respectively. The mediating effects account for approximately $56.13 \%$ of the total effect.

\subsection{Analysis of the moderating effect of resilience on social support and life satisfaction}

Structural Equation Modeling is used to test the moderating effect of resilience on social support and life satisfaction. Because it involves the interaction of latent variables, all indexes are centrally processed (Wen \& 
$\mathrm{Wu}$ 2010). The two social support indexes are multiplied by two psychological resilience indexes by means of index pairing. The product of two observation variables is obtained and used as the latent variable interaction index of social support and resilience. The mediation model is fitted and the fitting indexes of the model are: $X^{2} / \mathrm{df} \approx 12.114, \mathrm{GIF}=0.939$, $\mathrm{NFI}=0.912, \mathrm{CFI}=0.919, \mathrm{IFI}=0.919, \mathrm{RMSEA}=0.091$. Each index is above 0.9 and RESEA is less than 0.1 .

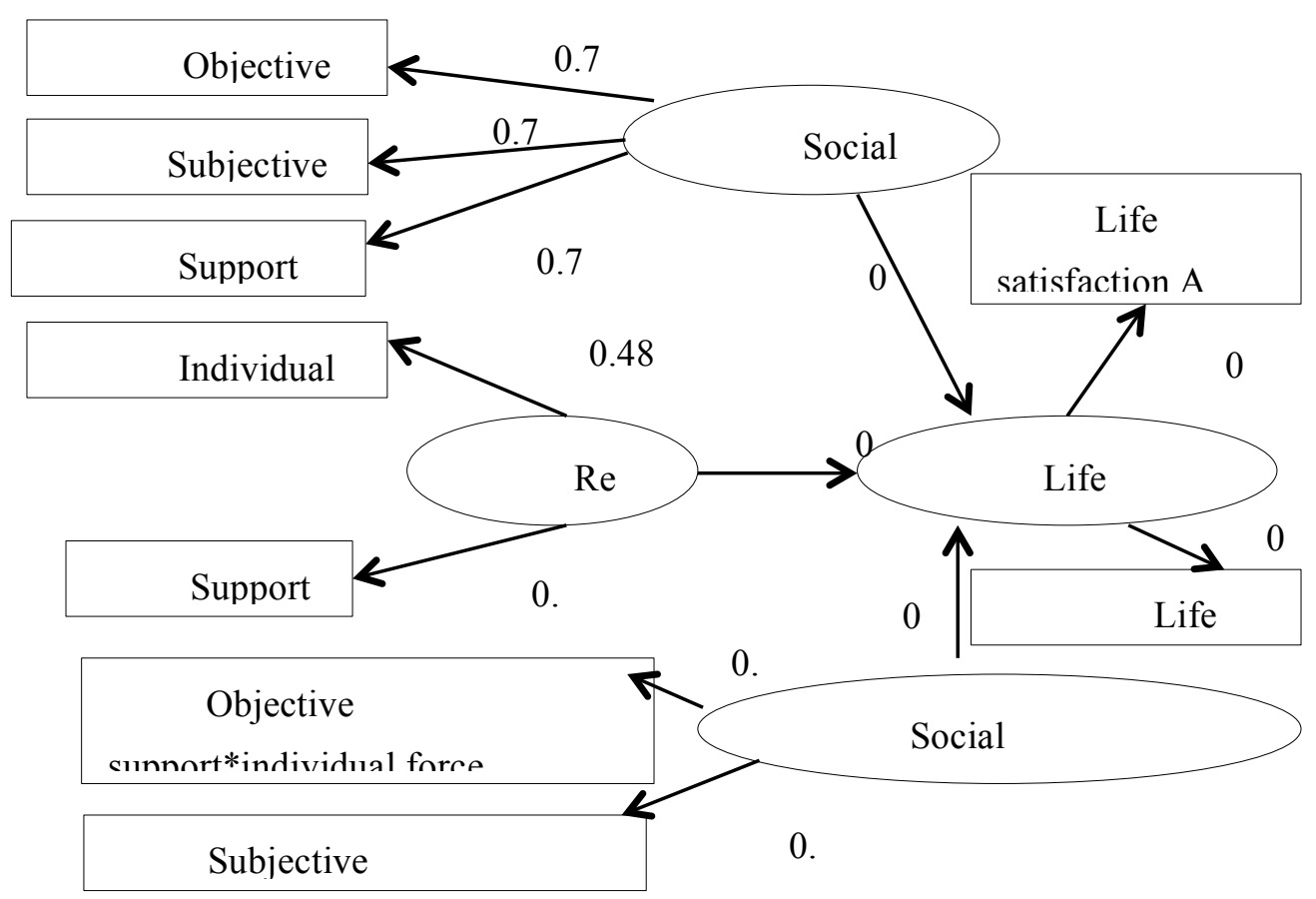

Figure 2. The path diagram of the moderating effect of resilience on social support and life satisfaction 


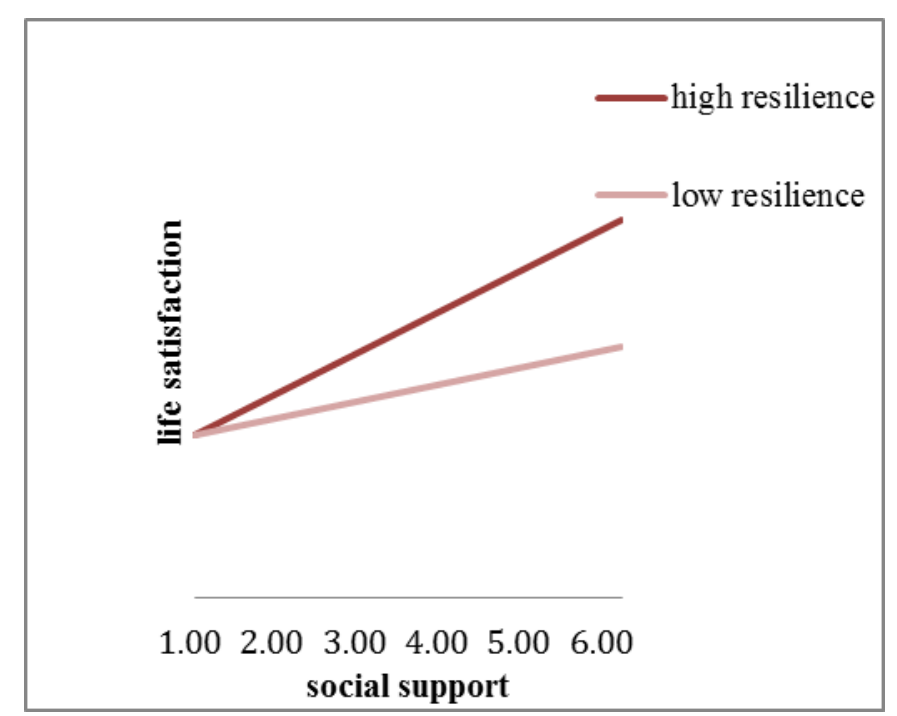

Figure 3. The moderating effect diagram of resilience on social support and life satisfaction

The results are shown in Figure 2. The path coefficients of social support, resilience, and social support*resilience to life satisfaction are all significant. The main effects are as follows: $0.342(\mathrm{P}<0.001), 0.878$ $(\mathrm{P}<0.001), 0.142(\mathrm{P}<0.01)$. This illustrates that the moderating effect of resilience on social support and life satisfaction is significant.

In order to further examine the influence of social support on life satisfaction of subjects with different resilience levels, the top $27 \%$ subjects with the highest resilience score are regarded as the high resilience group. The $27 \%$ subjects with the lowest resilience score of are regarded as the low resilience group. The relationship between social support and life satisfaction is shown in Figure 3. Resilience and social support have an interactive effect on life satisfaction, not a nonlinear relationship. There is a strong connection between social support and life satisfaction among subjects with high resilience. Among those with low resilience, social support also has a comparatively strong relationship with life satisfaction. However, the intensity of its relationship is less than with those with high resilience.

\section{Discussion}

\subsection{The mediating role of resilience on social support and life satisfaction}


Our study shows that social support and resilience of college students have a positive predictive effect on life satisfaction. This is consistent with previous studies (He \& Wang 2016; Xie \& Li 2014). The mediating effect shows that social support not only directly influences life satisfaction, but also indirectly influences it through resilience. The ratio of mediating effect to total effect is approximately 56\%. The results show that the positive effect of social support on life satisfaction is achieved with resilience as a mediator. Individuals with high social support tend to have higher support force and greater opportunities to improve their resilience (Song et al. 2014). Resilience, as an important psychological quality of individuals, can play a guiding role in individuals' life satisfaction (Liang et al. 2016). These results further support the notion that social support is a protective factor for individual mental health and that its positive effect on life satisfaction is partially achieved through individual resilience (Xie et al. 2014). In terms of mental health education, students' life satisfaction and their subjective well-being can be increased by improving their resilience.

\subsection{The moderating role of resilience on social support and life satisfaction}

Another finding in this study is that social support can play a more active role in life satisfaction. Individuals with high resilience can strengthen the positive effect of social support on life satisfaction. Individuals with high social support but low resilience are inclined to have lower life satisfaction compared to those with high resilience. Individuals with low social support but high resilience are likely to have higher life satisfaction than individuals with low resilience. The protective effect of social support is more obvious among individuals with high resilience than individuals with low resilience. This supports the protective factor model. (Li et al. 2009) The results of this study indicate that, in terms of mental health education for college students, we should consider not only increasing students' social support level, but also focusing on their resilience characteristics (Song et al. 2014).

\subsection{Enlightenment}


Our study indicates that resilience plays the role of mediation and moderation between social support and life satisfaction. Hence it can be concluded that development of resilience and enhancement of social support is of significance for improvement of life satisfaction. First, relevant departments of the school can regularly invite domestic and foreign experts to hold academic lectures discussing topics such as self-cognition, growth, and development. This would enhance students' self-cognition, self-consciousness, and emotional management ability. Additionally, this will cultivate students' resilience and enhance their perception. Second, schools should regularly conduct group counseling, build a practical platform for mental health education, introduce teachers, set up related courses, and promote college students' perception, experience and internalization of positive resilience. Third, a social support network comprising society, family and school should be formed. College students in the "Sturm und Drang" period are under the pressure of the present and have a longing for the future. Attention and support from their school, family and society enable them to have a sense of identity, belonging and trust (Li \& Yin 2015). College students will also be able to perceive the existence of subjective support from society, dispel current pressures, fears, and hesitations in order to improve their life satisfaction. Finally, students should be encouraged to actively participate in voluntary activities, to care for vulnerable groups, to play a positive guiding role, to learn to be grateful, and to enhance the positive experience of life.

\subsection{Limitations}

Although the sample size is large, the samples are all from Guangdong University of Foreign Studies. The limitations of this sampling method limit the extension of the results (John et al. 2010). In subsequent studies, samples covering more regions are needed for analysis and verification. There may also be a certain amount of error in the form of self-reporting by the subjects (Feng 2016). If other people's evaluations, behavior tracking observations, multiple records and reports are combined, the social desirability effect can be reduced. Finally, though it reveals the mediating and moderating effect of resilience on social support and life satisfaction, this study is designed for cross sectional 
study. The longitudinal study and design of the psychological mechanism of college students' life satisfaction will need to be considered in a subsequent research.

\section{Acknowledgments}

This paper is supported by National education science of China "the 13th Five-year Plan" 2016 program (approval number: EBA160407): Research on the Formation Mechanism of Pro-social Behaviors of College Students.

\section{References}

Chen K. \& Zhang Y. Y. 2013. "Study of Mediating Effect of Loneliness on Social Support and Subjective Well-Being of College Students." Journal of Chongqing University (Social Science Edition) 19 (4):172-181.

Cui L. Q. \& Yao B. X. 2012. "Relations Between Social Support and Life Satisfaction of New Generation Peasant-Workers." Journal of Hunan Agricultural University (Social Sciences) 8 (2):41-44.

Feng Zh. Y. \& Wan P. Y. 2016. "The Relationship Between Social Support, Resilience. Cyber-Bullying and Life Satisfaction Among College students." Chinese Journal of Health Education 32 (1):8-11.

Hu Y. Q. \& Gan Y. Q. 2008. "Development and Psychometric Validity of the Resilience Scale for Chinese Adolescents." Acta Psychologica Sinica 40 (8):902-12.

He H \& Wang Sh. 2016. "Social Support and Life Satisfaction of Young Migrants." Population Research 40 (3):45-57.

Xie J. Sh. \& Li. J. 2014. "Life Events and Life Satisfaction: Mediating Effect of Resilience in Junior High School Students." Chinese Journal of Clinical Psychology 22(4):676-679.

Li Q. 1998. "Social Support and Individual Mental Health." Tianjin Social Sciences 7 (1):67-70.

Li Y. X. \& Luo P. CH. 2009. "The Impact of Social Support on Resilience of College Students." Journal of Henan University (Social Science) 49(6):127-131. 
Liang S. C., Wu H. M., Yang Y. J., \& Yang D. 2016. "Mediating Effect of Mindfulness and Resilience on Relationship Between Neuroticism and Life Satisfaction in College Students." Chinese Mental Health Journal 30 (12):946-951.

John J. S., Eugene B. Z., \& Jeanne S. Z. 2010. "Research Methods in Psychology." Posts \& Telecom Press.

Zhang Y \& Xing Zh. J. 2007. "A General Overview of the Relationship Between Social Support and Subjective-Wellbeing." Psychological Science 30 (6):1436-8.

Li Zh. Y. 2010. "Relationship Between Social Support and Subjective Well-Being: Mediator Role of Self-Consistency and Congruence." Chinese Journal of Clinical Psychology 18(3):346-348.

Li Zh. H. \& Yin X.Y. 2015."How Social Support Influences Hope in College Students: The Mediating Roles of Self-Esteem and Self-Efficacy." Psychological Development and Education 31 (5):610-617.

Skoe E. A. 2010. "The Relationship Between Empathy Related Constructs and Care-based Moral Development in Young Adulthood." Journal of Moral Education 39(2):191-211.

Malti T., Eisenberg N., Kim H., \& Buchmann M. 2013. "Developmental Trajectories of Sympathy, Moral Emotion Attributions, and Moral Reasoning: The Role of Parental Support." Social Development 22(4):773-793.

Ma J. \& Wang Y. Zh. 2013. "The Mediating Effect of University Students' Social Support between Emotional Intelligence and Life Satisfaction." China Journal of Health Psychology 21(1):137-8.

Ou Y. L. 2017. "Research on the Relationship Between Resilience and Life Satisfaction Among College Students." China School Health 38(4):554-561.Self-e

Pietrzak R. H., Johnson D. C., \& Goldstein M. 2010. "Psychosocial Buffers of Traumatic Stress, Depressive Symptoms, and Psychosocial Difficulties in Veterans of Operations Enduring Freedom and Iraqi Freedom: The Role of Resilience, Unit Support, and Post Deployment Social Support." Journal of Affective Disorders 120:188-192.

Pavot W. G., Diener E., Colvin C. R., \& Sandvik E. 1991. "Further Validation of the Satisfaction with Life Scale: Evidence for the Cross-Method 
Convergence of Well-Being Measures." Journal of Personality Assessment 57:149-161.

Ratelle C. F., Simard K., \& Guay F. 2013. "University Students' Subjective Well-being: The Role of Autonomy Support from Parents, Friends, and the Romantic Partner." Journal of Happiness Studies 7 (14) 3:893-910.

Sun Sh. X., Guan Y. H., Qin Y. Y., Zhang L., \& Fan F. 2013. "Social Support and Emotional-Behavioral Problems: Resilience as a Mediator and Moderator." Chinese Journal of Clinical Psychology 2:114-118.

Tsui A. S., Ashford S. J., Clair L., \& Xin K. R. 1995. "Dealing with Discrepant Expectations: Response Strategies and Managerial Effectiveness." Academy of Management Journal 38 (6):1515-43.

Song G. W., Luo F., \& Zhou F. F. 2014. "On the Relationship Between the Social Support of Students with Outstanding Academic Performance and Students with Learning Difficulties Their Resilience and Mental Health." Chinese Journal of Special Education 165 (3):48-53.

Wang Y. \& Wang Zh. H. 2013. "Resilience of College Students and the Relations of Resilience to Positive Emotion and to Subjective Well-Being." Psychological Development and Education 1:94-100.

Wang Y. \& Wang Zh. H. 2014. "Cognitive Reappraisal and Satisfaction with Life: Multiple Mediating of Affect and Resilience." Chinese Journal of Clinical Psychology 22 (2):306-314.

Wu Y. \& Wen Zh. L. 2011. "Item Parceling Strategies in Structural Equation Modeling." Advances in Psychological Science 19 (21):1859-67.

Wen Zh. L. \& Wu Y. 2010. "Evolution and Simplification of the Approaches to Estimating Structural Equation Models with Latent Interaction." Advances in Psychological Science 18 (8):1306-13.

Wang X. K., Li L. Zh., \& Mu S. K. 2016. "The Effect of Social Support on Adolescents' Life Satisfaction: A Moderated Mediating Model." Journal of Minnan Normal University 12 (30):107-113.

Wang L. Ch. 2014. Relationship Among Psychological Capital, Social Support, and Degree of Satisfaction Campus Life in College Students. Retrieved from http://cdmd.cnki.com.cn/Article/CDMD-106811015004162.htm on August 7, 2018. 
$\mathrm{Wu}$ M. L. 2010. A Structural Equation Model - Manipulation and Application of AMOS. Chongqing: Chongqing University Press.

Xiao Sh. Y. 1994. "The Theoretical Basis and Research Application of the Social Support Rating Scale." Journal of Clinical Psychological Medicine 4(2):98-100.

Xie J. Sh. \& Deng Ch. L. 2014. "Social Support and Life Satisfaction: Mediating Effect of Resilience in Junior High School Students." Chinese Journal of Clinical Psychology 22(4):676-679.

Ye Y. Q. \& Lu Ai. T. 2017. "The Effect of Social Support on Learning Burnout of College Students: Moderated Mediation Model of Financial Condition and Life Satisfaction". Psychological Research 10(4):79-85.

Yu J. H., Li D., \& Wang F. X. 2013. "Relationship Among Social Support, Coping Styles and Life Satisfaction of Armed Police Recruits." Psychological Research 1:71-74.

Yang X. F. 2009. "A Study on the Relationship Among Social Support, Core Self-evaluation and Subjective Well-being in College Students." Chinese Journal of Special Education 114 (12):84-91.

Adela Y., Noreen E. M., \& Thomas J. Y. 2001. "Social Support and Well-Being in Early Adolescents." The Role of Mediating Variables 10 (2):163-181.

Baron R. M. \& Kenny D. A. 1986. The Moderator-Mediator Variable Distinction in Social Psychological Research: Conceptual, Strategic, and Statistical Considerations." Journal of Personality \& Social Psychology 51:1173-82.

Fredrickson B. L., Cohn M. A., Coffey K. A., Pek J., \& Finkel S. M. 2008. "Open Hearts Build Lives: Positive Emotions, Induced Rhrough Loving-kindness Meditation, Build Consequential Personal Resources." Journal of Personality and Social Psychology 95:1045-62. 


\section{Yuan Guo (Guangdong) \\ Relationship Between Social Support and Life Satisfaction of College \\ Students: Resilience As a Mediator and Moderator}

Abstract: A total of 843 college students in Guangdong University of Foreign Studies were investigated using a social support evaluation scale, a resilience scale, and a life satisfaction evaluation scale. Results were analyzed using IBM ${ }^{\circledR}$ SPSS $21 .{ }^{\circledR}$ and Amos 17.0. It was shown that there existed a positive correlation respectively among resilience, social support and life satisfaction. Social support predicted resilience positively and resilience partially mediated the association between social support and life satisfaction. Finally, resilience moderated the association between social support and life satisfaction; the higher the resilience level, the more significant the positive predictive effect of social support on life satisfaction. College students' life satisfaction is closely related to social support and resilience; resilience partially plays a mediating and moderating role between social support and life satisfaction.

Key words: Social support, life satisfaction, resilience, well-being, Chinese college students, mediator, moderator

Ethics in Progress (ISSN 2084-9257). Vol. 8 (2017). No. 2, Art. \#3, pp. 28-43.

Creative Commons BY-SA 3.0

Doi: $10.14746 /$ eip.2017.2.3 